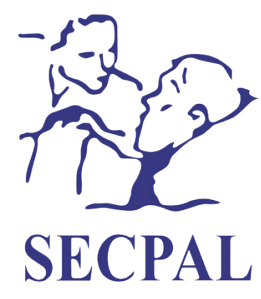

Medicina Paliativa

www.medicinapaliativa.es

ORIGINAL

\title{
Actitudes ante la muerte en voluntarios acompañando pacientes al final de la vida: estilos de apego y fatiga por compasión
}

\author{
José Carlos Bermejoํㅜ, Xavier Azcoitia ${ }^{2}$, Marisa Magaña ${ }^{3}$, Hadil Hassoun ${ }^{1}$ y Marta Villacieros ${ }^{* 1}$ \\ ${ }^{1}$ Centro de Humanización de la Salud, Tres Cantos, Madrid. España. ${ }^{2}$ Responsable de Atención Espiritual y del Voluntariado. \\ Centro San Camilo. Madrid. ${ }^{3}$ Coordinadora del Centro de Escucha. Centro San Camilo. Madrid. España
}

Recibido el 16 de mayo de 2017

Aceptado el 16 de julio de 2017

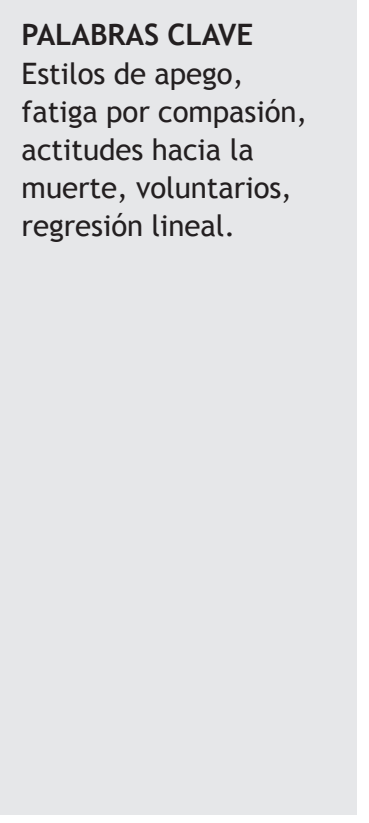

\begin{abstract}
Resumen
Objetivo: Evaluar las actitudes hacia la muerte en voluntarios, negativas (evitación, aceptación de escape y miedo a la muerte) y positivas (aceptación neutral y acercamiento), teniendo en cuenta los estilos de apego y la fatiga por compasión.

Método: La muestra estaba compuesta por 89 voluntarios de un centro sociosanitario, con una edad comprendida entre los 24 y los 81 años. Se llevó a cabo una regresión lineal para predecir las actitudes hacia la muerte, tanto positivas como negativas, partiendo de las variables calidad de vida profesional, satisfacción y fatiga por compasión, estilos de apego, edad, situación laboral, creencias religiosas, creencias en el más allá.

Resultados: La actitud positiva hacia la muerte obtuvo una $\mathrm{R}^{2}$ de 0,424 , siendo las variables en la regresión creencias en el más allá $(\beta=0,369, p<0,001)$, creencias religiosas $(\beta=0,272$, $\mathrm{p}<0,001)$, edad $(\beta=0,195, \mathrm{p}<0,029)$ y situación laboral $(\beta=-0,186, p<0,032)$. La actitud negativa hacia la muerte obtuvo un $\mathrm{R}^{2}=0,118$, siendo la única variable incluida en la ecuación el apego preocupado $(\beta=0,347, \mathrm{p}<0,001)$.

Conclusión: Se establece un modelo capaz de predecir el 42 \% de la varianza con respecto a las actitudes positivas hacia la muerte, y un $12 \%$ hacia las actitudes negativas. Según se avanza en el nivel de creencias religiosas, creencias sobre el encuentro en más allá y edad, y cuanto menor es el tiempo de ocupación laboral se encuentra actitud más positiva hacia la muerte. Sin embargo, la actitud negativa hacia la muerte se define por un estilo de apego preocupado.
\end{abstract}

\footnotetext{
*Autor para correspondencia:

Marta Villacieros Durbán

Centro de Humanización de la Salud. Sector Escultores 39, Tres Cantos, Madrid, España

Correo electrónico: investigacion@humanizar.es
} 


\section{KEYWORDS}

Attachment styles, compassion fatigue, attitudes toward death, volunteers, linear regression.

\section{Abstract}

Objective: To evaluate the attitudes toward death in volunteers; negative (fear of death, avoidance and escape acceptance) and positive attitudes (approach acceptance and neutral acceptance), considering attachment styles and compassion fatigue.

Method: The sample included 89 volunteers of a healthcare center, between 24 and 81 years-old. Multiple regression analysis were used to predict attitudes towards death, so much positive as negative, departing from the variables, professional quality of life, compassion satisfaction and fatigue, attachment styles, age, employment situation, religious beliefs and afterlife beliefs.

Results: Positive attitudes toward death showed $\mathrm{R}^{2}=0.424$, being included in regression equation the variables afterlife beliefs $(\beta=0.369, p<0.001)$, religious beliefs $(\beta=0.272, p<0.001)$, age $(\beta=0.195, p<0.029)$ and employment situation $(\beta=-0.186, p<0.032)$. Negative attitudes toward death showed $\mathrm{R}^{2}=0.118$, being the only variable included in regression equation concerned attachment style $(\beta=0.347, p<0.001)$.

Conclusion: A model predicting the $42 \%$ of the variance with respect to positive death attitudes and $12 \%$ to negative attitudes has been set. According to advancing the level of religious beliefs, afterlife beliefs and age, and the lower is the time of occupation, positive attitude towards death are developed. However the negative attitude toward death is defined by a concerned attachment style.

Bermejo JC, Azcoitia X, Magaña M, HAssoun H, Villacielos M. Actitudes ante la muerte en voluntarios acompañando pacientes al final de la vida: estilos de apego y fatiga por compasión. Med Paliat. 2019;26(1):55-61.

\section{Introducción}

El papel que desempeñan los voluntarios en las unidades de cuidados paliativos (UCP) es importante, ya que estos forman parte del servicio sanitario ofreciendo un apoyo social y psicológico a los pacientes y sus familiares ${ }^{1}$. Aun así, los voluntarios tienen un alto riesgo de padecer diversas secuelas debido a la exposición de situaciones que exigen una demanda emocional alta, pudiendo generar estados emocionales negativos, dolorosos y estresantes, como sucede en el caso de cuidar a pacientes al final de su vida².

Sobre el perfil de los voluntarios y sus modos de afrontamiento se encuentran estudios centrados en aspectos como la motivación, las experiencias previas con la muerte o la personalidad $^{3-6}$. Un estudio realizado por Walshe y cols. ${ }^{7}$ evalúa las repercusiones negativas para los voluntarios que trabajaban en la UCP, encontrado así niveles altos de estrés cuando fallecen sus pacientes. Las estrategias de afrontamiento que se encontraron en estos 400 voluntarios iban desde el distanciamiento con el paciente hasta mantener una fe religiosa y asumir que la muerte es un fin y no implica solo dolor ${ }^{8}$.

También entre los profesionales sociosanitarios, al estar en contacto con sus pacientes, se encuentran repercusiones como la evitación hacia aspectos de su trabajo y el miedo a la muerte o la "reacción emocional a la percepción de señales de peligro o amenaza, ciertas o imaginadas de la propia existencia, que pueden desencadenarse ante estímulos ambientales, situacionales, y estímulos internos de la persona relacionados con la propia muerte o ajena".

Para evaluar las actitudes hacia la muerte se han desarrollado diversos instrumentos como la escala de Collet-Lester $^{10}$, la escala de Bugen ${ }^{11}$, el Cuestionario de Actitudes ante la Muerte $(C A M-1)^{12}$ y el Perfil Revisado de Actitudes hacia la Muerte $(\mathrm{PAM}-\mathrm{R})^{13}$. Así, en las enfermeras se han encontrado actitudes negativas hacia el cuidado de sus pacientes cuan- to mayor era el miedo a la muerte ${ }^{14}$ que, por otro lado, se relaciona con problemas de salud, medidas de hipocondría, niveles altos de ansiedad y estrés y actitudes de evitación en el contexto profesional ${ }^{15}$.

Como se observa, el miedo a la muerte puede derivar en un tipo de estrés ya definido en la literatura como fatiga por compasión $^{16}$, resultante de la relación de ayuda terapéutica, que se define como un estado de tensión y preocupación extremos en aquellos que ayudan a otros, pudiendo constituir un evento traumático y que es derivado del desgaste por empatía ${ }^{17}$. Sus niveles se miden con el cuestionario de Calidad de Vida Profesional, Satisfacción y Fatiga por Compasión (Professional Quality of Life Scale Compassion Satisfaction and Fatigue, ProQOL$(V-R)^{18}$. En relación a esta dimensión se ha prestado atención a las variables individuales de los profesionales sociosanitarios, siendo la personalidad el componente principalmente relacionado con la fatiga ${ }^{19}$, los mecanismos de afrontamiento ${ }^{20}$, el género ${ }^{21}$, las creencias religiosas y la edad ${ }^{22}$.

Actualmente, también los estilos de apego han ido cobrando una gran importancia en el área de investigación del miedo a la muerte. El apego adulto, según Bowlby ${ }^{23}$, se constituye a partir de las relaciones tempranas y determinará las capacidades de las personas para afrontar el estrés y la regulación de las emociones en un contexto social. Ainsworth, Blehar, Waters y Wall ${ }^{24}$ desarrollaron la primera clasificación de apego en niños y describieron tres patrones generales: seguro, inseguro evitativo y ambivalente.

Existen diversos estudios que han demostrado la asociación entre los estilos de apego y la autoeficacia, autoestima, dependencia a sustancias, ansiedad, estrés, empatía, competencia interpersonal, psicopatologías, etc. ${ }^{25-28}$. Los estudios que se han centrado en su relación con el trabajo han encontrado asociación del apego inseguro con la falta de capacidad empática y con reacciones negativas ${ }^{29}$. Al contrario, las personas con estilo de apego seguro parecen estar 
satisfechos en sus trabajos, establecen buenas relaciones y muestran bienestar general ${ }^{30}$.

Respecto al estilo ambivalente, sujetos en este estilo reportan niveles más altos de afecto negativo en el trabajo como malestar, culpa, miedo y nerviosismo ${ }^{31}$. En estudiantes ${ }^{32}$ se ha encontrado asociación inversa entre burnout y apego seguro, y directa entre burnout y apego ambivalente y evitativo.

Por último, un estudio realizado por Mikulincer y Florian ${ }^{33}$ concluye que las personas con rasgo de apego ambivalente muestran niveles altos de miedo y pensamientos recurrentes hacia la muerte, mientras que las personas con un apego evitativo muestran actitud evitativa ante la muerte.

Como vemos, es importante conocer las actitudes ante la muerte de los voluntarios, su posible origen y consecuencias. Aun así, existen pocos estudios desarrollados en nuestro país y menos en el ámbito de las UCP, que pongan en relación esta variable, con los estilos de apego y la fatiga por compasión. Dependiendo del estilo de apego (seguro o no) y de la satisfacción (o fatiga) desarrollada en la acción de voluntariado, es posible que se generen actitudes más o menos favorables hacia la muerte. Por esta razón, el objetivo de este estudio fue analizar la relación entre estas tres variables en una muestra de voluntarios de una Unidad de Cuidados Paliativos.

\section{Metodología}

\section{Diseño}

El estudio consistió en un diseño descriptivo correlacional y transversal.

\section{Participantes}

Se recogieron 89 cuestionarios de un total de 114 voluntarios que participaban en el acompañamiento psicológico y emocional, mediante la escucha, a personas en procesos de duelo o crisis vitales. El centro sociosanitario concertado de la Comunidad de Madrid en el que ejercen su labor de voluntariado incluye una Residencia Asistida, una Unidad de Cuidados Paliativos y un Centro de Formación. Todos los voluntarios reciben una formación básica relativa al acompañamiento y sobre el perfil de personas al que van a atender.

En la reunión anual de voluntarios, se ofreció a los 114 asistentes participar en el estudio, teniendo en cuenta que, para ello, era necesario tener experiencia de voluntariado de al menos seis meses en el centro. Se recogieron 89 cuestionarios y se excluyeron dos por contener más de un $50 \%$ de valores perdidos, por lo que la muestra final quedó en 87 cuestionarios, siendo la tasa de respuesta del $78 \%$.

\section{Instrumentos}

Se elaboró un cuestionario ad hoc para recoger información sobre los siguientes aspectos:

- Variables sociodemográficas: sexo, edad, formación, situación laboral, actitud religiosa, creencias sobre la muerte, experiencia previa y actual como voluntariado (tiempo dedicado en años y meses, centro de voluntariado [asis- tencial o escucha], tiempo que le dedica a la semana y relación entre su rol de voluntariado y su profesión).

- También, se recogió la satisfacción con el voluntariado en cuanto a los aspectos organizativos y el contenido del voluntariado, en una escala del 1 (nada satisfecho) al 10 (totalmente satisfecho).

- Por último, se incluyó el tiempo que se dedica a sí mismo (en horas al mes) y su satisfacción con este tiempo dedicado a si mismo (del 1 [nada satisfecho] al 10 [totalmente satisfecho]).

Para medir las actitudes hacia la muerte se utilizó la versión española del Perfil Revisado de Actitudes hacia la muerte (PAM$\mathrm{R})^{13}$, el cual consta de 32 ítems y respuesta tipo Likert (del 1 al 5), que diferencia cinco tipos de actitudes, las tres primeras se pueden considerar negativas y las dos últimas positivas:

- Miedo a la muerte o respuesta consciente ante la muerte, temor a la pérdida de sí mismo, a lo desconocido, al más allá de la muerte, al dolor y al sufrimiento y el bienestar de los miembros supervivientes de la familia.

- Evitación ante la muerte o rechazo al tema de la muerte y toda situación, diálogo, lugar, que se asocie con la misma.

- Aceptación de escape en personas sin habilidades de afrontamiento eficaz para enfrentar el dolor o los problemas de la existencia, por lo que la muerte se puede convertir en una alternativa y puede ofrecer el único escape ante las emociones negativas.

- Aceptación neutral o ambivalencia presente ante la muerte, temor normal ante la muerte, aceptación ante la posible llegada como hecho innegable en la vida y aprovechamiento de la vida que se tiene.

- Aceptación de acercamiento, relacionada con creencias religiosas, donde se tiene una mayor confianza y está presente la creencia de una vida feliz después de la muerte.

Se calculó la fiabilidad mediante consistencia interna en la muestra del estudio $(n=87)$, obteniendo un alfa de Cronbach igual a 0,83 .

Para medir las posibles consecuencias se utilizó la escala Fatiga de Compasión y Satisfacción PROQOL-IV-R (Professional Quality of Life Scale) en su versión española18. La medida está formada por 30 ítems en total, con respuesta tipo Likert, que va desde el 0 (nunca) al 5 (siempre), e incluye en su medida tres dimensiones:

- La satisfacción por compasión o el placer que uno obtiene por ser capaz de hacer bien su trabajo.

- El burnout o sentimientos de desesperación y dificultades para afrontar el trabajo.

- El trauma secundario o fatiga por compasión, debido a la exposición a sucesos traumáticos en el trabajo.

La consistencia interna obtenida en la muestra del estudio fue un alfa de Cronbach del 0,85.

Para valorar los estilos de apego de los voluntarios se utilizó la escala CAMIR-R (Cartes Modeles Individuels the Relation) ${ }^{34}$, que mide las representaciones de apego. En su versión reducida en castellano ${ }^{35}$ incluye 32 ítems con una respuesta tipo Likert de 5 puntos ( 1 = totalmente en desacuerdo, 5 = totalmente de acuerdo) y distingue siete dimensiones:

- El apego seguro estaría recogido en la dimensión seguridad (o disponibilidad y apoyo de las figuras de apoyo). - El apego evitativo en la dimensión autosuficiencia (o rechazo de sentimientos de dependencia y reciprocidad afectiva y rencor hacia los seres queridos). 
El apego preocupado en las dimensiones preocupación familiar (o percepción de una intensa ansiedad de separación de los familiares y preocupación por las figuras de apego), e interferencia de los padres (o sobreprotección en la infancia, haber sido miedoso y sentimientos de preocupación por abandono).

El apego desorganizado en la dimensión traumatismo infantil (o haber experimentado falta de disponibilidad, violencia y amenazas).

La consistencia interna obtenida en los participantes del estudio según alfa de Cronbach fue de 0,78.

\section{Procedimiento}

Enmarcado en una reunión anual formativa para voluntarios, a todos los asistentes (un total de 114) se les entregaron los cuestionarios indicándoles las instrucciones y dándoles el tiempo de respuesta necesario que resultó entre 20 y 30 minutos. Previamente se les explicó el objetivo de la investigación, la voluntariedad de participar y la confidencialidad de los resultados de la misma.

\section{Análisis de datos}

Se calculó la consistencia interna de las escalas y subescalas mediante alfa de Cronbach con el programa estadístico Factor 10.4. Se utilizaron los coeficientes de correlación de Pearson para obtener la relación entre las variables cuantitativas de estudio y se realizó un análisis de regresión múltiple por pasos (stepwise) para determinar aquellas variables que pudieran predecir las actitudes tanto positivas como negativas hacia la muerte comprobando previamente los supuestos de la regresión múltiple (normalidad, homocedasticidad, no colinealidad, independencia y linealidad). Por último, se llevó a cabo una T de Student para comparar las medias de dos muestras independientes. Se empleó el paquete estadístico SPSS 22.

\section{Resultados}

\section{Características sociodemográficas}

De los 87 voluntarios, 67 eran mujeres (77\%) y 20 hombres (23\%). La media de edad fue de 54,92 años (DT = 13,29, min. = 24, máx. =81). En la Tabla I se detallan las distribuciones de formación (la mayoría licenciados), la situación laboral (la mayoría trabajadores con jornada completa $[27,6] \%$ y parcial $[11,5 \%]$, y la mayoría $(71,3 \%)$ realizando su actividad en el centro de escucha. En cuanto a la actitud religiosa, más de la mitad son creyentes practicantes (católico) y la gran mayoría cree que más allá de la muerte nos reencontramos con Dios.

Respecto a la experiencia en voluntariado, la media del tiempo total dedicado previamente fue de 113,69 meses $(D T=133,83)$ o 9,5 años (DT $=11,15)$, mientras que del tiempo en el propio centro fue de 46,49 meses (DT $=45,54$ ) o 3,8 años (DT $=3,79)$. El tiempo a la semana dedicado al voluntariado alcanza una media de 4,07 horas (DT $=3,58$ )
Tabla I. Variables sociodemográficas

\begin{tabular}{|c|c|c|}
\hline Variables $(n=87)$ & Frecuencia & Porcentaje \\
\hline \multicolumn{3}{|l|}{ Sexo: } \\
\hline Mujer & 67 & 77 \\
\hline Hombre & 20 & 23 \\
\hline \multicolumn{3}{|l|}{ Formación: } \\
\hline ESO o bachillerato & 7 & 8 \\
\hline Formación profesional & 2 & 2,3 \\
\hline Diplomatura & 14 & 16,1 \\
\hline Licenciatura o superior & 64 & 73,6 \\
\hline \multicolumn{3}{|l|}{ Situación laboral: } \\
\hline Jornada completa & 24 & 27,6 \\
\hline Jornada parcial & 10 & 11,5 \\
\hline Desempleado & 15 & 17,2 \\
\hline Jubilado & 29 & 33,3 \\
\hline Estudiante & 4 & 4,6 \\
\hline Otro & 5 & 5,7 \\
\hline \multicolumn{3}{|l|}{ Actitud religiosa: } \\
\hline $\begin{array}{l}\text { Creyente practicante } \\
\text { (católico) }\end{array}$ & 49 & 56,3 \\
\hline $\begin{array}{l}\text { Creyente no practicante } \\
\text { (católico) }\end{array}$ & 15 & 17,2 \\
\hline Agnóstico/ateo & 17 & 19,5 \\
\hline Otra religión & 6 & 6,9 \\
\hline \multicolumn{3}{|l|}{ Creo que más allá del morir: } \\
\hline No hay nada & 11 & 12,6 \\
\hline $\begin{array}{l}\text { Nos reencontramos con } \\
\text { nuestros seres queridos }\end{array}$ & 15 & 17,2 \\
\hline Nos reencontramos con Dios & 61 & 70,1 \\
\hline \multicolumn{3}{|l|}{ Centro de voluntariado: } \\
\hline Centro asistencial & 25 & 28,7 \\
\hline Centro de escucha & 62 & 71,3 \\
\hline
\end{tabular}

y la satisfacción con el voluntariado en cuanto a los aspectos organizativos y el contenido del voluntariado alcanza unas medias de $8,13(D T=1,49)$ y $8,77(D T=1,24)$ sobre 10 puntos. El tiempo dedicado a sí mismo una media de $36,68$ horas al mes (DT $=29,69)$ y, por último, la satisfacción con el tiempo dedicado a sí mismo presenta una media de 7,65 (DT = 1,87) sobre 10 puntos.

En la Tabla II podemos observar las correlaciones entre las variables cuantitativas del estudio. Se encontró relación significativa de la actitud negativa hacia la muerte con el apego evitativo $(0,239, p<0,05)$ y con el apego preocupado $(0,358, p<0,01)$. También se encontró relación significativa de la actitud positiva hacia la muerte, con el apego evitativo $(0,278, p<0,001)$ y con la edad $(0,397, p<0,05)$. 
Tabla II. Correlaciones entre variables cuantitativas

\begin{tabular}{|c|c|c|c|c|c|}
\hline Variables & A. Seguro & A. Evitativo & A. Preocupado & A. Desorganizado & Edad \\
\hline $\begin{array}{l}\text { A. Positiva } \\
\text { muerte }\end{array}$ & 0,132 & $0,278^{* *}$ & 0,128 & 0,041 & $0,397^{* *}$ \\
\hline $\begin{array}{l}\text { A. Negativa } \\
\text { muerte }\end{array}$ & 0,106 & $0,239^{*}$ & $0,358^{* *}$ & 0,059 & 0,010 \\
\hline
\end{tabular}

Ninguna de las dimensiones del cuestionario PROQOL-IV, mostró correlación con ninguna de las dimensiones de actitud hacia la muerte, es decir, no se encuentra correlación significativa entre fatiga por compasión y actitud negativa hacia la muerte.

Para estudiar el carácter predictivo de las dimensiones que componen el CAMIR-R y el PROQOL-IV, así como las variables creencias religiosas, creencias en el más allá, situación laboral y edad respecto a las actitudes hacia la muerte (positivas y negativas), se realizó un análisis de regresión múltiple por pasos.

Respecto al cumplimiento de los supuestos, la prueba de Kolmogorov-Smirnov para actitud positiva y negativa hacia la muerte resultó no significativa ( $p>0,05)$, por lo que se verifica el cumplimiento del supuesto de normalidad. El supuesto de homocedasticidad, mediante el estadístico Levene, se cumple en ambas variables $(p>0,05)$. El estadístico de Durbin-Watson toma un valor igual a 1,89 en la variable actitud positiva y un valor de 1,94 en actitud negativa, aproximándose al 2 en ambas, por lo que los residuos son completamente independientes. Por último, el supuesto de no colinealidad se calculó mediante el nivel de Tolerancia (Tabla III); en todos los casos se aproxima a 1 para actitud positiva, mientras que para actitud negativa es igual a 1,00, por lo tanto, para ambas variables se cumple dicho supuesto.

La variable actitud positiva hacia la muerte obtuvo una $R^{2}$ de 0,424 , por lo que se puede decir que las variables finalmente incluidas en la regresión (Tabla III) creencias en el más allá, creencias religiosas y situación laboral, predicen el 42,4 \% de la actitud positiva hacia la muerte. Para todas las variables se obtuvieron coeficientes beta positivos, excepto para la situación laboral, lo que quiere decir que según se avanza en el nivel de creencias religiosas, creencias sobre el encuentro en más allá y edad, se encuentra actitud más positiva hacia la muerte; sin embargo, cuanto menor es el tiempo de ocupación laboral las actitudes hacia la muerte son más positivas.

Para la variable actitud negativa hacia la muerte, se obtiene una $R^{2}=0,118$, siendo la única variable incluida en la ecuación el apego preocupado. Esto indica que esta dimensión logra explicar el $12 \%$ de la variabilidad de la actitud negativa hacia la muerte. El coeficiente de regresión beta fue de 0,347 ( $p<0,001)$.

Tampoco en esta prueba se encuentra relación alguna entre fatiga por compasión y actitudes hacia la muerte.

\section{Discusión}

Este estudio analiza la relación existente entre los estilos de apego, la fatiga por compasión y la actitud hacia la muerte (positiva o negativa) en los voluntarios de un centro sociosanitario. Para ello se utilizan cuestionarios validados en castellano con amplio recorrido tanto en investigación como en clínica ${ }^{13,18,35}$ y se aportan datos de consistencia interna comprobando su fiabilidad en esta muestra. Así, los resultados obtenidos apoyan la hipótesis de que el estilo de apego preocupado tiene poder predictivo sobre las actitudes negativas hacia la muerte (miedo, evitación y escape), pero no sostiene la hipótesis de la existencia de relación entre fatiga por compasión y actitudes hacia la muerte.

Tabla III. Coeficientes de regresión múltiple para la variable actitud positiva hacia la muerte

\begin{tabular}{|c|c|c|c|c|c|c|c|c|}
\hline Variables incluidas & $\mathbf{R}^{2}$ & $\mathbf{R}^{2}$ corregida & $F$ (sig.) & $\beta$ & $\mathrm{t}$ & $\mathrm{p}$ & IC $95 \%$ & Tolerancia \\
\hline Paso 4 & 0,451 & 0,424 & $\begin{array}{l}160,61 \\
(0,000)\end{array}$ & & & & & \\
\hline $\begin{array}{l}\text { Creo en el más } \\
\text { allá del morir }\end{array}$ & & & & 0,369 & 30,82 & 0,000 & 30,87 a 120,25 & 0,730 \\
\hline Creencias religiosas & & & & 0,272 & 20,88 & 0,005 & 10,51 a 80,24 & 0,766 \\
\hline Edad & & & & 0,195 & 20,21 & 0,029 & 0,023 a 0,434 & 0,874 \\
\hline Situación laboral & & & & $-0,186$ & $-20,18$ & 0,032 & $-110,24$ a $-0,533$ & 0,937 \\
\hline
\end{tabular}

$R^{2}$ : coeficiente de determinación. $F$ (sig.): estadístico $F$ (nivel de significación). $\beta$ : coeficiente de regresión. $t=T$ de Student . p: nivel de significación. IC $95 \%$ : intervalo de confianza del $95 \%$. 
Resultados similares se han encontrado en el estudio realizado por $\mathrm{Caras}^{36}$, que demostró una relación significativa entre el miedo a la muerte, ansiedad por separación y estilos de apego, siendo los niveles de ansiedad hacia la muerte bajos cuando las personas tenían un estilo de apego seguro, y altos cuando presentaban un estilo de apego preocupado. Las personas con estilo de apego preocupado se mantienen hipervigilantes a las fuentes de peligro, mostrando actitud de escape, miedo y evitación. De acuerdo con Bowly ${ }^{37}$, los estilos de apego mantienen la proximidad con otras personas significantes y protegen al ser humano de las amenazas tanto físicas como simbólicas, entre las que se encuentra la muerte.

Otro hallazgo de este estudio se encuentra en las variables actitud religiosa y creencias en el más allá, ya que ambas se relacionan de manera significativa con las actitudes positivas hacia la muerte, siendo las personas creyentes y las que creen en un reencuentro con Dios las que muestran actitud de aceptación neutral y de acercamiento. Este hallazgo está en la línea de otros estudios ${ }^{38}$ donde se encuentran correlaciones positivas entre creencias religiosas y la aceptación de la muerte; quienes manifiestan ser creyentes, practicantes y con mayor grado de religiosidad expresan menos miedo a la muerte ${ }^{39}$.

Asimismo, cuanto menor es el tiempo de ocupación laboral las actitudes hacia la muerte son más favorables. En este estudio la mayoría de los voluntarios trabajadores son sociosanitarios, que ya están expuestos en su día a día a sucesos traumáticos y muerte. Según otros estudios, ${ }^{40}$, los trabajadores sociosanitarios están más predispuestos a tener actitudes de miedo hacia la muerte debido al fallecimiento de las personas con las que trabajan, que enfrenta a los profesionales a sus propios miedos, ansiedades y temores ${ }^{41}$. Este hecho podría estar enmascarando algunos resultados.

Entre nuestros resultados observamos que los voluntarios de mayor edad tienen menos actitudes negativas hacia la muerte, en la línea de lo que también se ha encontrado en otros estudios, en los que los jóvenes tenían mayor miedo hacia la muerte que los adultos ${ }^{42}$.

Entre las limitaciones que encontramos en este estudio está el hecho de que los participantes no hayan sido seleccionados por procedimientos aleatorios y/o en diferentes centros, razón por la que encontramos un perfil profesional poco variado. A esto hay que sumar el hecho de que existen múltiples variables relacionadas con el miedo a la muerte, recoger medidas de la gran variabilidad existente es un gran reto para poder establecer relaciones causales.

Aunque sabemos que la experiencia de cercanía con la muerte puede generar una situación de demanda emocional que, en el caso de los voluntarios de centros sociosanitarios, es importante considerar, lo que al final se destaca en este estudio es que se deben considerar también variables personales e individuales de los propios voluntarios, como los estilos de apego y las creencias religiosas, que muestran un gran poder explicativo.

Las actitudes de los voluntarios en el acompañamiento de pacientes son importantes para el manejo psicoemocional adecuado de los enfermos ya que, en alguna medida, puede condicionar incluso la actitud de los propios pacientes ante su enfermedad y la muerte. Es importante favorecer el desarrollo de actitudes adecuadas ante situaciones de muerte. En definitiva, en los equipos multidisciplinarios se da el abordaje de esta situación en pacientes y familiares y la formación y el seguimiento del voluntariado es un elemento fundamental para que el apoyo prestado pueda contribuir al objetivo de mejorar la calidad de vida en situaciones de complejidad para pacientes y cuidadores.

\section{Bibliografía}

1. Claxton-Oldfield S, Claxton-Oldfield J. The impact of volunteering in hospice palliative care. Am J Hosp Palliat Care. 2007;24(4):259-63.

2. Kearney MK, Weininger RB, Vachon ML, Harrison RL, Mount BM. Self-care of physicians caring for patients at the end of life: "Being connected... a key to my survival". Jama. 2009;301(11):1155-64.

3. Bermejo JC, Villacieros M, Magaña M. Perfil, motivación, satisfacción y compromiso de un voluntariado de acompañamiento en duelo. Propiedades psicométricas de los cuestionarios Organizational Commitment Questionnarie y Job Diagnostic Survey. Medicina Paliativa. 2014;24(1):4-13.

4. Mitchell C, Shuff I. Personality characteristics of hospice volunteers as measured by the Myers Briggs Type indicator. J Pers Assess. 1995;65(3):521-32.

5. Geiser C, Okun MA, Grano C. Who is motivated to volunteer? A latent profile analysis linking volunteer motivation to frequency of volunteering. Psychol Test Assess Model. 2014;56(1):3-24.

6. Vantilborgh T, Bidee J, Pepermans, Willems J, Huybrechts G, Jegers $M$. Revisiting the relationship between personality and psychological contracts: a moderated mediation model explaining volunteer performance. Soc Serv Rev. 2013;87(1):158-86.

7. Walshe C, Dodd S, Hill M, Ockenden N, Payne S, Preston N, et al. How effective are volunteers at supporting people in their last year of life? A pragmatic randomised wait-list trial in palliative care (ELSA). BMC Med. 2016;14(1):203.

8. Dein S, Syed QA. The stresses of volunteering in a hospice: A qualitative study. Palliat Med. 2005;19(1):58-64.

9. Uribe-Rodríguez AF, Valderrama L, Durán Vallejo DM, GaleanoMonrroy C, Gamboa K. Diferencias evolutivas en la actitud ante la muerte entre adultos jóvenes y adultos mayores. Acta Colombiana de psicología. 2008;11(1):119-26.

10. Collet L, Lester D. The fear of death and the fear of dying. $J$ Psychol. 1969;72(2):179-81.

11. Bugen LA. Coping: Effects of death education. J Death Dying. 1980-1981;11:175-83.

12. Grau J, Chacón M, Llantá MC, Infante O, Guerra ME, Barbat I, et al. Validación de una metodología para modificar actitudes ante la muerte en profesionales de la salud. Protocolo de Proyecto Citma (en curso); 2007.

13. Wong P, Rever G, Gesser T. Perfil Revisado de Actitudes ante la Muerte: un instrumento multidimensional. En: Neimeyer R (compilador). Métodos de evaluación de la Ansiedad ante la Muerte. Barcelona: Paidós; 1997. p.131-58.

14. Espinoza M, Sanhueza O. Miedo a la muerte y su relación con la inteligencia emocional de estudiantes de enfermería de Concepción. Acta paulista de enfermagem. 2012;25(4):607-13.

15. Noyes R, Stuart S, Langbehn DR, Happel RL, Longley SL, Yagla SJ. Childhood antecedents of hypochondriasis. Psychosomatics. 2002;43(4):282-9.

16. Limonero JT. El fenómeno de la muerte en la investigación de las emociones. Rev Psicol Gen Apl. 1996;49(2):249-65.

17. Figley CR. Compassion fatigue: Coping with secondary traumatic stress disorder. New York: Brunner/Mazel; 1995.

18. Stamm BH. 'The ProQOL Manual'. [Consultado 18 Oct 2015]. Disponible en: http://www.proqol.org/uploads/ProQol_vIV_ Spanish_Oct05.pdf . Adaptado por Morante ME, Moreno B, Rodríguez A. Traducción del Cuestionario de Fatiga de Compasión y Satisfacción (Cuarta Revisión); 2006. 
19. Swider BW, Zimmerman RD. Born to burnout: A meta-analytic path model of personality, job burnout and work outcomes. J Vocat Behav. 2010;76(3):487-506.

20. Bush NJ. Compassion fatigue: are you at risk? Oncol Nurs Forum. 2009;36(1):24-8.

21. Bayés R, Limonero JT, Buendía B, Burón E, Enríquez N. Evaluación de la ansiedad ante la muerte. Medicina Paliativa. 1999;6(4):140-3.

22. Edo-Gual M, Tomás-Sábado J, Aradilla-Herrero A. Miedo a la muerte en estudiantes de enfermería. Enferm Clin. 2011;21(3):129-35.

23. Bowlby J. Developmental psychiatry comes of age. Am J Psychiatry. 1988;145(1):1-10.

24. Ainsworth MDS, Blehar MC, Waters E, Wall S. Patterns of Attachment: a psychological study of the Strange Situation. Hillsdale, New Jersey: Erlbaum; 1978.

25. Dykas MJ, Cassidy J. Attachment and the processing of social information across the life span: theory and evidence. Psychol Bull. 2011;137(1):19-46.

26. Cole-Detke H, Kobak R. Attachment processes in eating disorder and depression. J Consult Clin Psychol. 1996;64(2):282-90.

27. Fonagy $P$, Leigh $T$, Steele $M$, Steele $H$, Kennedy R, Mattoon G, et al. The relation of attachment status, psychiatric classification, and response to psychotherapy. J Consult Clin Psychol. 1996;64(1):22-31.

28. Rosenstein DS, Horowitz HA. Adolescent attachment and psychopathology. J Consult Clin Psychol. 1996;64(2):244-53.

29. Cassidy J, Shaver PR. Handbook of attachment. New York: Guilford Press; 2008.

30. Hazan C, Shaver PR. Love and work: An attachment-theoretical perspective. J Pers Soc Psychol. 1990;59(2):270-80.

31. Kerr S, Melley A, Travea L, Pole M. The relationship of emotional expression and experience to adult attachment style. Individ Differ Res. 2003;1(2):108-23.

32. Pines $A M$. Adult attachment styles and their relationship to burnout: A preliminary cross-cultural investigation. Work Stress. 2004;18(1):66-80.
33. Mikulincer M, Florian V. Exploring individual differences in reactions to mortality salience: Does attachment style regulate terror management mechanisms. J Pers Soc Psychol. 2000;79(2):260-73. [Consultado 5 Abr 2017]. Disponible en: https: / /www.researchgate.net/publication/292407923_Attachmentrelated_defensive_processes

34. Pierrehumbert B, Karmaniola A, Sieye A, Meisler C, Miljkovitch $R$, Halfon O. Les modeles de relations: Développement d'un autoquestionnaire d'attachement pour adultes. Psychiatr Enfant. 1996;39(1):161-206.

35. Balluerka N, Lacasa F, Gorostiaga A, Muela A, Pierrehumbert B. Versión reducida del cuestionario CaMir (CaMir-R) para la evaluación del apego. Psicothema. 2011;23(3):486-94.

36. Caras GW. The relationships among psychological separation, the quality of attachment, separation anxiety and death anxiety. Dissertation Abstracts International Section B: The Sciences and Engineering. 1995;56(6):3436.

37. Bowlby J. Attachment and loss: Separation. New York: Basic Books; 1973.

38. Harding SR, Flannelly KJ, Weaver AJ, Costa KG. The influence of religionon death anxiety and death acceptance. Ment Health Relig Cult. 2005;8(4):253-61.

39. Tomás-Sábado J, Limonero JT. Religiousness and death anxiety. En: Ambrose SD, editor. Religion and Psychology: New Research. New York: Nova Science Publishers; 2006. p. 107-22.

40. Grau J, Llantá MC, Massip C, Chacón M, Reyes MC, Infante O, et al. Ansiedad y actitudes ante la muerte: revisión y caracterización en un grupo heterogéneo de profesionales que se capacita en cuidados paliativos. Pensamiento psicológico. 2008;4(10):27-58.

41. Figueredo Borda N. Prácticas de cuidado y percepciones de maltrato en personas mayores institucionalizadas. [Tesis doctoral]. Barcelona: Universidad de Barcelona; 2015.

42. Edo-Gual M, Tomás-sábado J, Aradilla-Herrero A. Miedo a la muerte en estudiantes de enfermería. Enferm Clin. 2011;21(3):129-35. 